\title{
Hypocalcemia is highly prevalent and predicts hospitalization in patients with COVID-19
}

\author{
Luigi Di Filippo ${ }^{1}$ Anna Maria Formenti ${ }^{1}$ Patrizia Rovere-Querini ${ }^{2} \cdot$ Michele Carlucci $^{3} \cdot$ Caterina Conte $^{2}$. \\ Fabio Ciceri ${ }^{2} \cdot$ Alberto Zangrillo $^{4}$ - Andrea Giustina ${ }^{1}$
}

Received: 21 May 2020 / Accepted: 3 June 2020 / Published online: 12 June 2020

(c) Springer Science+Business Media, LLC, part of Springer Nature 2020

Coronavirus disease 2019 (COVID-19), caused by the Severe Acute Respiratory Syndrome Coronavirus 2 (SARSCoV-2), presents primarily with fever, dry cough, and fatigue or myalgia [1]. Although most patients have a favorable prognosis, infection may not infrequently lead to a severe syndrome requiring hospitalization and assisted ventilation with high lethality [2].

Previous studies have reported that calcium played a central role in viral infectious and replicative mechanisms of SARS-CoV, MERS-CoV, and Ebolavirus [3-5]. In a large group of SARS patients in North America, hypocalcemia was detected in $60 \%$ of patients at hospital admission and in $70 \%$ during hospitalization [6]. Moreover, data from patients with Ebolavirus infection in United States and European hospitals reported a similar incidence of hypocalcemia [7].

Several studies investigated so far the clinical and laboratory characteristics of COVID-19 patients, including inflammatory and organ injury biomarkers [8]. Recently, we have reported the first case of COVID-19 presenting with severe hypocalcemia [9]. However, no population data are available on calcium levels in COVID-19 [10].

Andrea Giustina

giustina.andrea@hsr.it

1 Department of Endocrinology, Vita-Salute San Raffaele University and IRCCS San Raffaele Scientific Institute, Milan, Italy

2 Vita-Salute San Raffaele University and Division of Transplantation, Immunology and Transplantation Diseases, IRCCS San Raffaele Scientific Institute, Milan, Italy

3 Emergency Department, IRCCS San Raffaele Scientific Institute, Milan, Italy

4 Department of Anesthesia and Intensive Care, Vita-Salute San Raffaele University and IRCCS San Raffaele Scientific Institute, Milan, Italy
The aim of our study was to investigate the incidence of hypocalcemia in a large single center population of COVID19 patients and evaluate its possible clinical implications.

\section{Methods}

We conducted a retrospective cohort study at IRCCS San Raffaele Hospital, a tertiary health-care hospital in Milan, Italy. We included patients (aged $\geq 18$ years) with COVID19 admitted to our Emergency Department (ED). We excluded COVID-19 patients transferred from other hospitals and patients initially hospitalized for other diseases.

Only patients with serum ionized calcium data from arterial blood gas tests performed at initial evaluation in the ED were included. Ionized calcium levels were expressed both as actually measured levels (AC) and as adjusted mathematically to a standardized $\mathrm{pH}$ of 7.4. levels (SC) to avoid influence of sample handling. Hypocalcemia was defined as calcium level below $1.18 \mathrm{mmol} / \mathrm{L}$ (RapidPoint 500 Analyzer, VA, USA).

We excluded patients with comorbidities and concomitant therapies influencing calcium metabolism: chronic kidney disease, osteoporosis, patients on glucocorticoids and antiepileptic drugs, vitamin $\mathrm{D} /$ calcium, loop/thiazide diuretics, and patients with an eGFR $\leq 30 \mathrm{~mL} / \mathrm{min} / 1.73 \mathrm{~m}^{2}$ using creatinine levels at initial evaluation. Assessed outcomes included hospitalization, intensive care unit (ICU) admission and death.

This study is part of the COVID-19 Biobank study, which is registered with ClinicalTrials.gov, NCT04318366 and obtained specific approval by the local EC.

Statistical analysis was conducted with SPSS 23.0 version (Chicago, USA). Categorical variables were indicated as frequency (\%) and continuous variables as medians (IQR).

Differences in variable frequencies between groups were calculated using the Fisher test and the Mann-Whitney $U$ test for continuous variables. All statistical tests were two-sided. A $p$ value of $<0.05$ was considered statistically significant. 
Table 1 Baseline characteristics of patients with Covid-19 included in the study

\begin{tabular}{|c|c|}
\hline & No. $(\%)$ \\
\hline \multicolumn{2}{|l|}{ Demographic information } \\
\hline Total No. & 531 \\
\hline Age, median (IQR), year & $59(50-69)$ \\
\hline \multicolumn{2}{|l|}{ Gender } \\
\hline Female & $171(32.2 \%)$ \\
\hline Male & $360(67.8 \%)$ \\
\hline \multicolumn{2}{|l|}{ Ethnicity } \\
\hline Caucasian & $449(84.6 \%)$ \\
\hline Latin American & $70(13.2 \%)$ \\
\hline Asian & $8(1.5 \%)$ \\
\hline Sub-Saharan African & $4(0.8 \%)$ \\
\hline BMI, median (IQR), kg/m² & $27(24-30)$ \\
\hline \multicolumn{2}{|l|}{ Comorbidities } \\
\hline Total No. & 531 \\
\hline Hypertension & $177(33.3 \%)$ \\
\hline Coronary artery disease & $34(6.4 \%)$ \\
\hline \multicolumn{2}{|l|}{ Diabetes } \\
\hline Type 1 & $5(0.9 \%)$ \\
\hline Type 2 & $68(12.8 \%$ \\
\hline Cancer $^{\mathrm{a}}$ & $11(2.1 \%)$ \\
\hline Chronic obstructive pulmonary disease & $10(1.8 \%)$ \\
\hline Neurological disabilities & $11(2.1 \%)$ \\
\hline \multicolumn{2}{|l|}{ Laboratory results at admission } \\
\hline $\mathrm{pH}$, median $(\mathrm{IQR})$ & $7.47(7.44-7.5)$ \\
\hline \multicolumn{2}{|c|}{ Ionized calcium levels, median (IQR), mmol/L } \\
\hline Actual calcium & $1.1(1.07-1.15)$ \\
\hline Standardized calcium & $1.14(1.1-1.17)$ \\
\hline eGFR, median (IQR), mL/min/1.73 m² & $85(68.8-97.7)$ \\
\hline $\begin{array}{l}\text { Total calcium levels }{ }^{\mathrm{b}}, \text { median } \\
(\mathrm{IQR}), \mathrm{mmol} / \mathrm{L}\end{array}$ & $2.14(2.05-2.2)$ \\
\hline LDH, median (IQR), U/L & $359(273-457)$ \\
\hline CRP, median (IQR), mg/L & $66(26.2-123.4)$ \\
\hline
\end{tabular}

BMI body mass index; COVID-19 coronavirus disease 2019, IQR interquartile range

${ }^{\mathrm{a}}$ Only active neoplasms were included in this section

${ }^{\mathrm{b}}$ Calcium values at admission have not been corrected for serum albumin

Linear regression analyses were used to correlate continuous variables. Univariate and multivariate analyses performing binary logistic regression were used to calculate adjusted odds ratios (ORs) with 95\% confidence intervals (CIs).

\section{Results}

A total of 531 patients were included in the study. Demographic and clinical patients' characteristics are summarized in Table 1. On initial hospital evaluation, median AC level was $1.1 \mathrm{mmol} / \mathrm{L}$ [1.07-1.15] and SC level was $1.14 \mathrm{mmol} / \mathrm{L}$ [1.1-1.17]. Hypocalcemia was found in 462 patients $(82 \%)$ with $\mathrm{AC}$ levels, in 414 (78.6\%) patients with SC levels. Using AC levels 18 patients (3.4\%) presented a severe hypocalcemia with values lower than $0.99 \mathrm{mmol} / \mathrm{L}$, using SC this was found in 10 patients (1.9\%).

Hypocalcemic patients were more frequently males (AC, $69 \%$ vs $57 \% p=0.06$; SC, $70 \%$ vs $60 \% p=0.046$ ) and older (AC, 59 years [51-69] vs 53 years [45-67] $p=0.01$ ). LDH and CRP levels were very significantly higher in hypocalcemic vs normocalcemic patients (Fig. 1). Moreover, linear regression analyses showed a negative correlation of calcium levels with LDH (AC, $p<0.001$, $r^{2}=0.074$; SC, $\left.p<0.001, r^{2}=0.055\right)$ and CRP levels (AC, $p<0.001, r^{2}=0.038$; SC, $\left.p<0.001, r^{2}=0.025\right)$.

Four hundred twenty-four patients (79.8\%) were hospitalized after initial evaluation, they had significantly lower ionized calcium levels as compared to non-hospitalized patients (AC, 1.1 [1.06-1.4] vs 1.14 (1.1-1.18] mmoll/L, $p<0.001 ; \mathrm{SC}, 1.13$ [1.1-1.17] vs 1.16 [1.12-1.2] mmol/L, $p<0.001$ ). In univariate and multivariate analyses, hypocalcemia was an independent risk factor highly associated with hospitalization (AC, $p<0.001 \mathrm{SC}, p<0.001)$ (Table 2).

Thirty-four patients $(11.6 \%)$ and 33 patients $(11.5 \%)$, based on AC or SC levels, respectively, developed severe hypocalcemia during hospitalization.

Fifty-eight out of 531 patients died $(11.5 \%)$ and 62 $(11.7 \%)$ were admitted to ICU. Hypocalcemia at initial evaluation was significantly $(p<0.05)$ associated with these two outcomes only in univariate analyses (death: AC, OR 4.9 CI 95\% [1.18-20.8]; SC, OR 2.6 CI 95\% [1.1-6.32]; ICU admission: AC, OR 5 CI 95\% [1.19-20.9], SC, OR 2.7 CI 95\% [1.14-6.5]) but not in multivariate analyses.

\section{Discussion}

In previous studies, hypocalcemia was associated with higher mortality and poor clinical outcome in hospitalized and critically ill patients $[11,12]$.

Several reports demonstrated a crucial role of calcium in viral fusion for different enveloped viruses such as SARS-CoV, MERS-CoV, and Ebolavirus [3-5]. Calcium directly interacted with fusion peptides of these viruses promoting their replication [3-5]. Moreover, in SARS$\mathrm{CoV}$ and Ebolavirus patients, hypocalcemia was a very frequent laboratory finding although its mechanistic and clinical relevance as well as its prognostic significance were not fully elucidated $[6,7]$.

Surprisingly, so far despite the already extensive literature available in COVID-19 and its related biochemical markers of activity and prognosis, no peer reviewed 
Fig. 1 Inflammatory parameters in patients with (YES) or without (NO) hypocalcemia based on actual and standardized ionized calcium levels
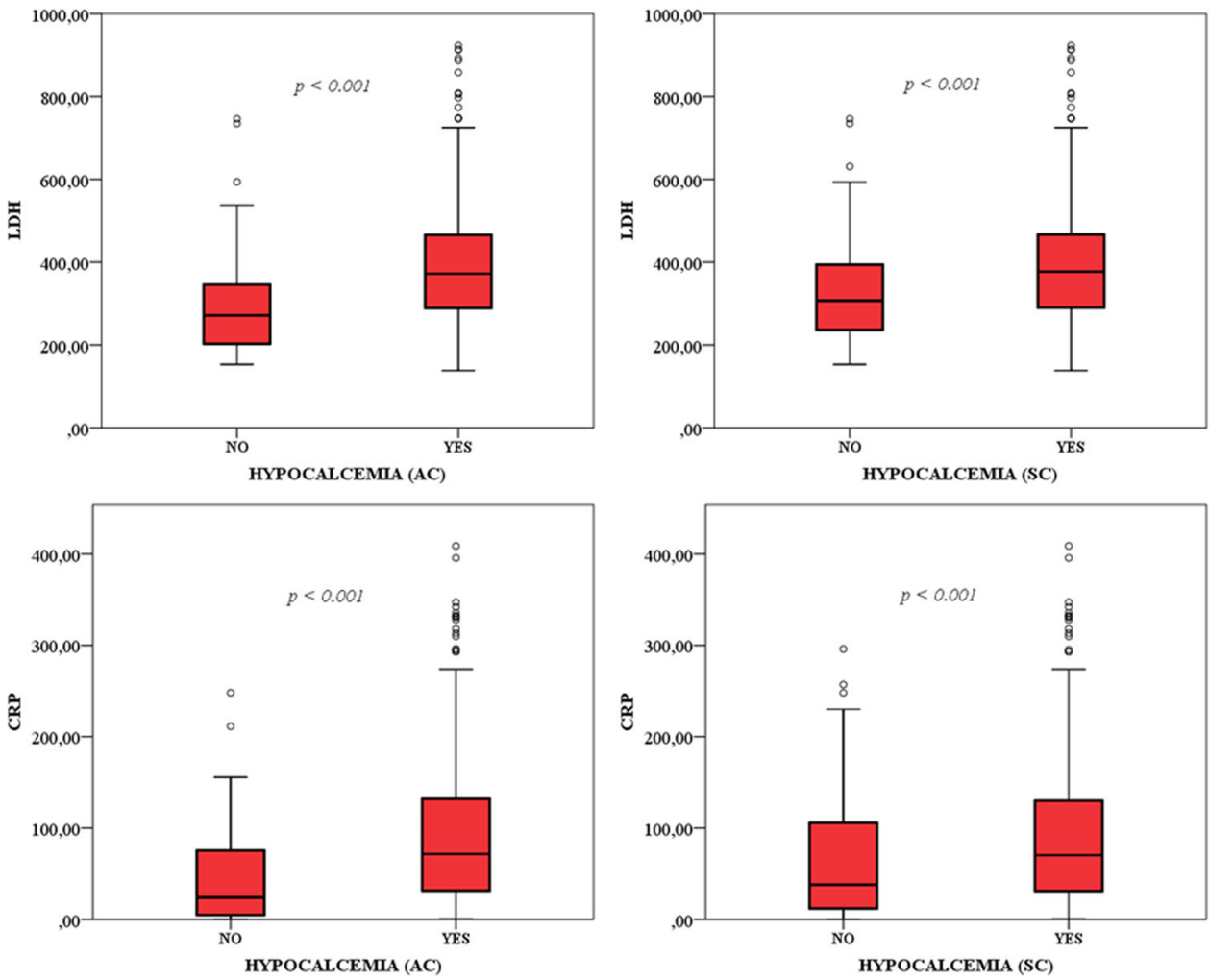

Table 2 Multivariate analyses of possible risk factors for hospitalization in our study population

\begin{tabular}{lcl}
\hline Variables* & $\begin{array}{l}\text { Odds ratio [95\% confidence } \\
\text { interval] }\end{array}$ & $p$ value \\
\hline A. Age & $1.06[1.03-1.09]$ & $\boldsymbol{p}<\mathbf{0 . 0 0 1}$ \\
Male gender & $1.83[1.002-3.35]$ & $\boldsymbol{p}=\mathbf{0 . 0 4 9}$ \\
Hypertension & $1.3[0.63-2.69]$ & $p=0.46$ \\
Coronary artery disease & $1.59[0.38-6.6]$ & $p=0.52$ \\
Type 2 diabetes & $2.24[0.75-6.6]$ & $p=0.15$ \\
Hypocalcemia (AC) & $2.27[2.72-11.6]$ & $\boldsymbol{p}<\mathbf{0 . 0 0 1}$ \\
eGFR & $1[0.99-1.02]$ & $p=0.42$ \\
LDH & $1.004[1.001-1.007]$ & $\boldsymbol{p}=\mathbf{0 . 0 0 4}$ \\
CRP & $1.008[1.002-1.014]$ & $\boldsymbol{p}=\mathbf{0 . 0 1 1}$ \\
B. Age & $1.06[1.03-1.09]$ & $\boldsymbol{p}<\mathbf{0 . 0 0 1}$ \\
Male gender & $1.67[0.91-3.0 .6]$ & $p=0.097$ \\
Hypertension & $1.23[0.59-2.52]$ & $p=0.57$ \\
Coronary artery disease & $1.83[0.45-7.47]$ & $p=0.4$ \\
Type 2 diabetes & $1.95[0.67-5.61]$ & $p=0.22$ \\
Hypocalcemia (SC) & $4.15[2.21-7.78]$ & $\boldsymbol{p}<\mathbf{0 . 0 0 1}$ \\
eGFR & $0.99[0.98-1.01]$ & $p=0.46$ \\
LDH & $1.005[1.002-1.008]$ & $\boldsymbol{p}=\mathbf{0 . 0 0 3}$ \\
CRP & $1.009[1.002-1.015]$ & $\boldsymbol{p}=\mathbf{0 . 0 0 6}$ \\
\hline
\end{tabular}

*Only variables with $p<0.3$ in univariate analyses were included in multivariate analyses. Section A: hypocalcemia based on AC; section B: hypocalcemia based on SC. $P$ values reported in bold are those statistically significant. published data are yet available on calcium levels in this emerging disease. Only recently we observed a patient with COVID-19 presenting with severe hypocalcemia who had in the history total-thyroidectomy as risk factor for subclinical hypoparathyroidism [13]. Therefore, to our knowledge this is the first study that revealed a very high incidence of hypocalcemia in a large monocentric population of COVID-19 patients at initial hospital evaluation. The observed rate of hypocalcemia was higher in comparison to the previous studies in SARS$\mathrm{CoV}$ and Ebolavirus patients and this could be explained partly because we used serum ionized calcium levels, a more reliable measure than total calcium levels corrected for albumin. Moreover, highly prevalent hypovitaminosis D in the northern regions of Italy may be a predisposing factor in our study population [14].

Calcium levels at initial evaluation were lower in finally hospitalized vs non-hospitalized patients, and hypocalcemia was found to be an independent risk factor for hospitalization. Moreover, the strong association between high LDH and CRP levels and low calcium levels which can be observed in Tumor Lysis Syndrome [15] may support the definition of COVID-19 as a Cytopathic Viral lysis syndrome. This suggests a possible role of ionized calcium as useful biochemical marker of disease aggressiveness, since also easy to measure in emergency, helping clinicians in identifying severe patients at initial hospital evaluation. 
Moreover, the relative high proportion of patients who developed severe hypocalcemia during hospitalization supports its specific relevance to the disease. In univariate analyses hypocalcemia was also found to be associated with mortality and ICU admission, but this was not maintained in multivariate analyses likely, due to many other interfering factors included different therapeutic approaches.

In conclusion, since hypocalcemia is highly incident in COVID-19 patients and predicts the need for hospitalization we suggest that ionized calcium should always be assessed at initial hospital evaluation in order to identify more severe patients. Finally, since hypocalcemia may have negative impact on cardiac outcomes and may be even lethal when severe and acute we suggest calcium monitoring and adequate supplementation when indicated in all hospitalized patients with COVID-19 [13].

\section{Data availability}

All authors had full access to all the data in the study and takes responsibility for the integrity of the data and the accuracy of the data analysis

Author contributions All authors contributed to the study conception and design. Material preparation, data collection and analysis were performed by L.D.F, A.M.F., P.R.Q., C.C. and A.G. The first draft of the manuscript was written by L.D.F., A.M.F. and A.G., and all authors commented on previous versions of the manuscript. All authors read and approved the final manuscript.

\section{Compliance with ethical standards}

Conflict of interest The authors declare that they have no conflict of interest.

Ethical approval All procedures performed in studies involving human participants were in accordance with the ethical standards of the institutional and/or national research committee and with the 1964 Helsinki declaration and its later amendments or comparable ethical standards.

Informed consent Written informed consent was waived.

Publisher's note Springer Nature remains neutral with regard to jurisdictional claims in published maps and institutional affiliations.

\section{References}

1. W.J. Guan, Z.Y. Ni, Y. Hu et al. Clinical characteristics of coronavirus disease 2019 in China. N. Engl. J. Med. 382(18), 1708-1720 (2020)

2. D. Wang, B. Hu, C. Hu et al. Clinical characters of 138 hospitalized patients with 2019 novel coronavirus-infected pneumonia in Wuhan, China. JAMA 323(11), 1061-1069 (2020)

3. J.K. Millet, G.R. Whittaker, Physiological and molecular triggers for SARS-CoV membrane fusion and entry into host cells. Virology 517, 3-8 (2018)

4. M.R. Straus, T. Tang, A.L. Lai et al. $\mathrm{Ca} 2+$ ions promote fusion of Middle East respiratory syndrome coronavirus with host cells and increase infectivity. J. Virol. pii: JVI.00426-20 (2020)

5. L. Nathan, A.L. Lai, J.K. Millet et al. Calcium ions directly interact with the Ebola virus fusion peptide to promote structurefunction changes that enhance infection. ACS Infect. Dis. 6(2), 250-260 (2020)

6. C.M. Booth, L.M. Matukas, G.A. Tomlinson et al. Clinical features and short-term outcomes of 144 patients with SARS in the Greater Toronto area. JAMA 289(21), 2801-2809 (2003)

7. T.M. Uyeki, A.K. Mehta, R.T. Davey Jr et al. Clinical management of Ebola virus disease in the United States and Europe. N. Engl. J. Med. 374(7), 636-646 (2016)

8. S. Richardson, J.S. Hirsch, M. Narasimhan et al. Presenting characteristics, comorbidities, and outcomes among 5700 patients hospitalized with COVID-19 in the New York City area. JAMA (2020). https://doi.org/10.1001/jama.2020.6775

9. Bossoni S., Chiesa L., Giustina A. Severe hypocalcemia in a thyroidectomized woman with Covid-19 infection. Endocrine (2020). https://doi.org/10.1007/s12020-020-02326-0

10. M. Puig-Domingo, M. Marazuela, A. Giustina, COVID-19 and endocrine diseases. A statement from the European Society of Endocrinology. Endocrine 68(1), 2-5 (2020)

11. W. Cheungpasitporn, C. Thongprayoon, M.A. Mao, W. Kittanamongkolchai, A. Sakhuja, S.B. Erickson, Impact of admission serum calcium levels on mortality in hospitalized patients. Endocr Res. 43(2), 116-123 (2018)

12. A. Akirov, A. Gorshtein, I. Shraga-Slutzky, I. Shimon, Calcium levels on admission and before discharge are associated with mortality risk in hospitalized patients. Endocrine 57(2), 344-351 (2017)

13. F. Tecilazich, A.M. Formenti, S. Frara, R. Giubbini, A. Giustina, Treatment of hypoparathyroidism. Best Pract Res Clin Endocrinol. Metab. 32(6), 955-964 (2018)

14. A. Giustina, A.M. Formenti, Preventing a covid-19 pandemic can high prevalence of severe hypovitaminosis $\mathrm{D}$ play a role in the high impact of Covid infection in Italy? BMJ 368, m810 (2020)

15. B. Rahmani, S. Patel, O. Seyam et al. Current understanding of tumor lysis syndrome. Hematol Oncol. 37(5), 537-547 (2019) 